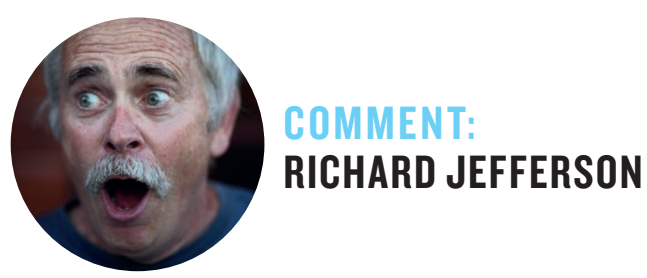

\title{
TURNING SCIENCE INTO SOCIAL OUTCOMES
}

\section{For innovation to bring public benefit, mapping the influence of academic papers is just the beginning.}

I $\mathrm{t}$ takes much more than science to make an impact on society. The complex nature of modern innovation requires assembling a jigsaw puzzle of complementary and essential capabilities before benefits reach the public as useful and affordable products. These include research findings, intellectual property, market and manufacturing knowledge, quality assurance, and legal standards, and regulatory compliance.

Finding, understanding, visualizing and assembling these capabilities is expensive and difficult. Knowledge lies in silos of specialization, curated and overseen by expensive, skilled, practitioners. These silos must be joined and mapped if the social and economic outcomes from science and technology are to be increased and the benefits more equitably distributed.

To develop maps of how science and technology influence industry, the idea of 'innovation cartography', the primary knowledge corpora need to be open, linked, standardized and made more meaningful. Openness is essential to achieve a network effect.

Patents are a critical part of the primary knowledge corpora. They are a main tool in the innovation strategy used by the private sector, but rarely by the public sector, to reveal findings and inventions that have anticipated economic use.

Because a patented invention must be publicly disclosed, patents offer a valuable insight into the actors, capabilities and aspirations of inventive product development. They also contain metadata links to diverse knowledge, including people and institutions, science, standards, legal and regulatory issues. Almost 50 million patent inventions disclosed in the last 100 years include aspects of virtually all technology in use today.

But, simply inferring the performance of the public sector by counting a university or scholar's patents or patent applications is of marginal use to determine or improve impact. In many cases, these patents are non-performing - most are never licensed - and mainly indicate the ambitions of the academic applicant, and the willingness of the university's commercialization office to fund the application in anticipation of licensing revenue. It is more often patents owned by third parties, informed by and citing academic work, that expose the influence of research on the development of products and services.

To map such influence on industry and the innovation process, we need to combine published research with the patent corpus.

When patents are filed, the applicants or the patent examiners often cite published scholarship that informs, contextualizes, influences or enables their new inventions. By linking these published citations to unambiguous identifiers, we can begin connecting the two bodies of knowledge, creating an influence

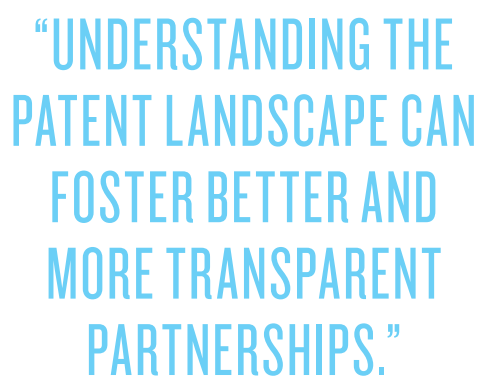

map. This would allow anyone to explore how published research findings and scholarship enable the process of innovation; and how the people and institutions interact to advance or hinder the uptake for society. This must occur in a free, open, private and secure format that encourages scientists and investors to discover and build bridges with the other actors in the innovation system.

Consider a disease vaccine whose lack of profitability means private industry alone cannot justify the investment and risks of commercialization.

This is a classic market failure. Effective disease prevention by immunization needs vastly more than the research that underlies discovery of the vaccine's immune target. All the other components are as important, such as demonstrating safety and efficacy through clinical trials and regulatory compliance. We have to know which institutions and what people should be 'at the table' to get the project done, and each needs to find sufficient incentive to participate and stay the course.

In this instance, open influence mapping would allow the academic research community and its public funders to better understand the complex innovation ecosystem. It can ensure the project operates by licensing or acquiring rights to third-party intellectual property - or inventing around it - from the outset.

Too often this process is tied up with research programmes conducted by academic scientists who neither know nor care about third-party intellectual property considerations. Understanding the patent landscape can foster better and more transparent partnerships.

Most innovative businesses spend vast sums to understand and map the knowledge and patent ecosystem in which they work. They cannot afford not to do it - the legal, business, financial and technical risks from ignorance are too great.

For the public sector, or for public-good targets, the same constraints and needs apply, but they cannot afford it, nor is it part of the culture of public science and investment. Yet it must be done.

The resolution is to make it cheaper, easier and more effective to join the knowledge corpora in a way that increases the quality of decisions for all participants, public and private, and enables more effective and equitable policy and practice of innovation.

Richard Jefferson is professor of Biological Innovation at Queensland University of Technology in Australia, and the founder of Cambia, a social enterprise, and Lens.org, a free, open and secure platform to make the innovation system more efficient, fair, transparent and inclusive. 